\title{
5-亚环己基-2-取代氨基咪唑啉酮类化合物的合成及其杀菌活性研究
}

\author{
雷建平韩金涛徐志红董宏波王明安* \\ (中国农业大学应用化学系 北京 100193)
}

\begin{abstract}
摘要 以环己酮和 2-硫代乙内酰嫝为起始原料, 经 Knoevenagel 缩合反应制得 5-亚环己基-2-硫代咪唑啉-4-酮(1), 化合 物 1 在乙醇钠/乙醇体系中与碘甲烷反应得到 5-亚环己基-2-甲硫基咪唑啉-4-酮(2), 化合物 2 再与相应的取代苯胺或苄 胺在冰醋酸体系中回流制得目标化合物 5-亚环己基-2-取代氨基咪唑啉酮 3a 3r, 它们的化学结构经 ${ }^{1} \mathrm{H}$ NMR, IR, MS 和 X-ray 单晶衍射确证. 5-亚环己基-2-对氯芐氨基咪唑啉酮(3q)： $M_{\mathrm{r}}=335.83, \mathrm{C}_{16} \mathrm{H}_{18} \mathrm{ClN}_{3} \mathrm{O} \cdot \mathrm{CH}_{4} \mathrm{O}$, Monoclinic, $P 2(1) / n$, $\rho=1.264 \mathrm{~g} / \mathrm{cm}^{3}, F(000)=712, Z=4, a=0.59895(12) \mathrm{nm}, b=1.2161(2) \mathrm{nm}, c=2.4289(5) \mathrm{nm}, \beta=94.03(3)^{\circ}$. 初步生物活性 测定结果表明: 在 $50 \mu \mathrm{g} / \mathrm{mL}$ 浓度下, 部分目标化合物均对供试菌种显现出一定的抑制活性，其中 5-亚环己基-2-对氟苄 氨基咪唑啉酮 (3p) 对油菜菌核的 $\mathrm{EC}_{50}$ 为 $24.37 \mu \mathrm{g} / \mathrm{mL}, \mathbf{3 q}$ 对辣椒疫霉的 $\mathrm{EC}_{50}$ 为 $28.68 \mu \mathrm{g} / \mathrm{mL}$.
\end{abstract}

关键词 5-亚环己基-2-氨基取代咪唑啉酮, 2-硫代乙内酰脲, Knoevenagel 缩合反应，杀菌活性

\section{Synthesis and Fungicidal Activity of 5-Cyclohexylidene-2- aminoimidazolin-4-one Derivatives}

\author{
Lei, Jianping Han, Jintao Xu, Zhihong Dong, Hongbo Wang, Mingan* \\ (Department of Applied Chemistry, China Agricultural University, Beijing 100193)
}

\begin{abstract}
A series of new 5-cyclohexylidene-2-aminoimidazolin-4-one derivatives 3a $\sim \mathbf{3 r}$ were synthesized via Knoevenagel condensation, methylation and substitution reaction using cyclohexanone and 2-thiohydantoin as starting material. Their structures of compounds $\mathbf{3 a} \sim \mathbf{3 r}$ were confirmed by ${ }^{1} \mathrm{H}$ NMR, IR, MS techniques and X-ray diffraction. 5-Cyclohexylidene2-p-chlorobenzylaminoimidazolin-4-one (3q): $M_{\mathrm{r}}=335.83, \mathrm{C}_{16} \mathrm{H}_{18} \mathrm{ClN}_{3} \mathrm{O} \cdot \mathrm{CH}_{4} \mathrm{O}$, Monoclinic, $P 2(1) / n, \rho=1.264 \mathrm{~g} / \mathrm{cm}^{3}$, $F(000)=712, Z=4, a=0.59895(12) \mathrm{nm}, b=1.2161(2) \mathrm{nm}, c=2.4289(5) \mathrm{nm}, \beta=94.03(3)^{\circ}$. The preliminary bioassay shows that some of the compounds have certain fungicidal activities at the concentration of $50 \mu \mathrm{g} / \mathrm{mL}$. The $\mathrm{EC}_{50}$ value of 5-cyclohexylidene-2-p-fluorobenzylaminoimidazolin-4-one (3p) against Sclerotinia scleotiorum is $24.37 \mu \mathrm{g} / \mathrm{mL}$, and that of 3q against Phytophthora capsici is $28.68 \mu \mathrm{g} / \mathrm{mL}$, respectively.

Keywords 5-cyclohexylidene-2-aminoimidazolin-4-one; 2-thiohydantoin; Knoevenagel reaction; fungicidal activity
\end{abstract}

咪唑啉酮衍生物是一类具有良好生物活性和药理 活性的杂环化合物，如 1998 年法国罗纳-普朗克-艾格 福公司在研究 2-甲硫基咪唑啉酮衍生物时, 发现该类化 合物表现出良好的杀菌活性，开发出第一个咪唑啉酮类 杀菌剂咪唑菌酮 I (RPA-407213) ${ }^{[1]}$, 它具有渗透、内吸 的特点, 表现出良好的保护和治疗活性, 尤其对由卵菌 引起的霜霉病、疫病等具有很好的活性, 对 I 的杀菌作 用机制的研究显示它是一种新型的线粒体呼吸抑制剂, 作用方式独特、杀菌谱广、尤对抗性菌株有效因而倍受
关注. 1999 年 Lacroix 等 ${ }^{[2]}$ 报道了 3-芐基咪唑啉酮 II, 在 $1 \mathrm{mg} / \mathrm{mL}$ 浓度时，对小麦锈病的防效大于 $75 \% .2002$ 年， Ewa 等 ${ }^{[3]}$ 合成了 5 -氯代芳基亚甲基-2-氨基取代咪唑啉4-酮衍生物 III, 并进行了体外抗结核分支杆菌、抗细菌 和抗真菌等生物活性测试，结果表明 2-氯和 2,4-二氯芳 基亚甲基乙内酰脲衍生物表现了较好的抗菌活性. 2004 年，丁明武等 ${ }^{[4,5]}$ 报道了具有 5-呋喃亚甲基咪唑啉䣶衍 生物 IV 和 V, 生物活性测定结果表明，化合物 IV 和 V 对稻瘟病、水稻纹枯病、芦笋褐斑病、苹果轮纹菌、小

\footnotetext{
*E-mail: wangma@cau.edu.cn

Received April 25, 2012; revised May 30, 2012; published online June 6, 2012.
}

Project supported by the National Key Technologies R\&D Program (No. 2011BAE06B04), the National Natural Science Foundation of China (No. 201172254) and the National Key Laboratory of Elemento-Organic Chemistry in Nankai University (Nos. 0902, 201003).

国家 “12.5” 科技支撑计划(No. 2011BAE06B04)、国家自然科学基金(No. 21172254)和南开大学元素有机化学国家重点实验室开放基金(Nos. 0902, 201003)资助项目. 
麦赤霉菌以及棉花枯萎菌均具有良好的抑制活性. 我们 研究小组 ${ }^{[6]}$ 发现, 含有环十二烷或酮的乙内酰脲衍生物 对芦笋茎枯、茄绵疫以及油菜菌核病菌均有优良的抑制 活性，抑制率在 70\% 98\%之间，其中 3 个化合物对芦 笋茎枯的 $\mathrm{EC}_{50}$ 值分别达到 $15.80,31.34$ 和 $44.26 \mu \mathrm{g} / \mathrm{mL}$, 而含有环十二烷或酩的硫代乙内酰脲衍生物的活性较 差. 目前尚未见有文献报道 5-亚环基取代的咪唑啉酮类 化合物的合成及其生物活性，因此在前期研究工作基础 上，为进一步提高化合物的生物活性，本文参考文献设 计合成了系列新化合物 3a 3r，以探讨 5-位取代基为亚 环基时其对咪唑啉酮衍生物杀菌活性的影响. 化合物的 合成路线如 Scheme 1 所示.
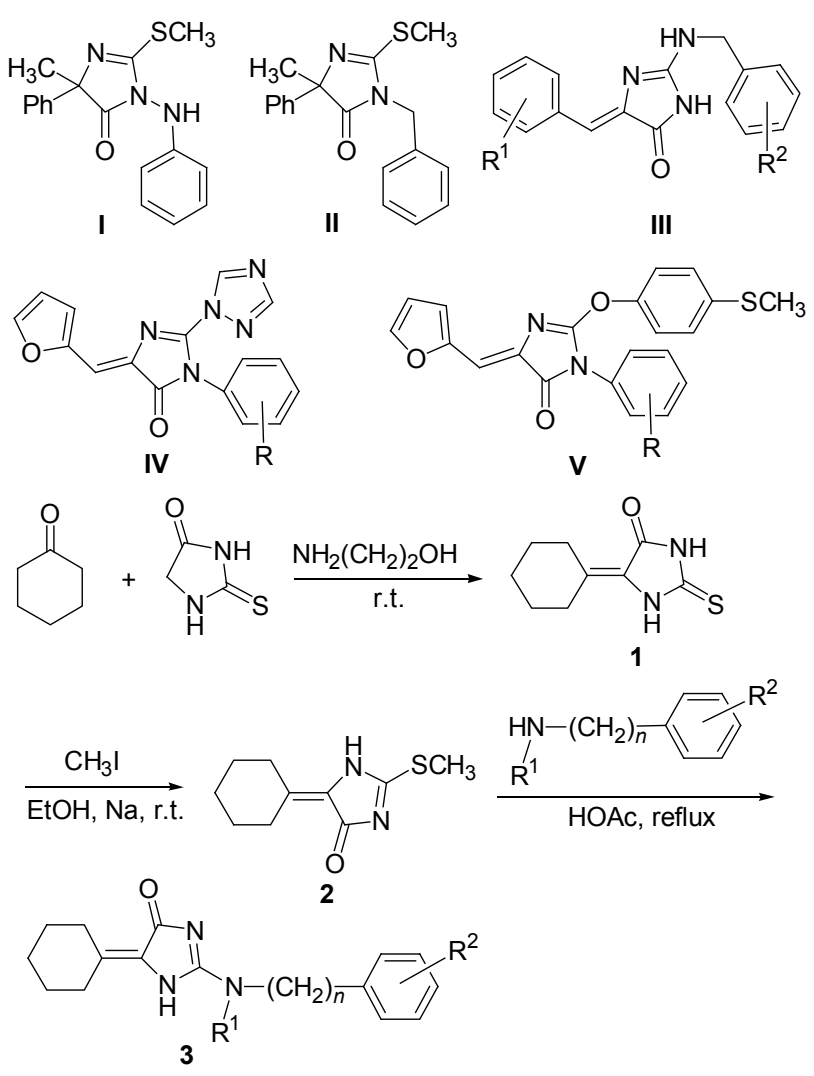

Scheme 1

\section{1 实验部分}

\section{1 仪器与试剂}

Cole-parmer 熔点仪(未校正); Bruker DPX 300MHz 核磁共振仪, 用 TMS 为内标, $\mathrm{CDCl}_{3}$ 或 DMSO- $d_{6}$ 为溶剂, 化学位移用 $\delta$ 表示; Perkin Elmer FTS-40 红外光谱仪 $\left(\mathrm{KBr}\right.$ 压片法), 单位为 $\mathrm{cm}^{-1}$. 本实验中所用试剂和溶剂 均由专业化学试剂公司提供的 AR 级试剂, 环己酮、取 代苯胺、取代芐胺分别购自国药集团化学试剂有限公司, Acros, J\&K Chemical, Matrix Scientific, TCI 等公司, 含 量均大于 $98 \%$.

\section{2 化合物的合成}

\subsubsection{2-硫代乙内酰脙的合成}

向 $100 \mathrm{~mL}$ 单口瓶中加入 $12.8 \mathrm{~g}(0.11 \mathrm{~mol})$ 甘氨酸, $7.6 \mathrm{~g}(0.10 \mathrm{~mol})$ 硫氰酸铵, $57.6 \mathrm{~mL}$ 乙酸酐和 $6.4 \mathrm{~mL}$ 冰醋 酸, 将体系置于 $100{ }^{\circ} \mathrm{C}$ 油浴中加热、搅拌, $40 \mathrm{~min}$ 后, 反 应完全，冷却，倒入 $300 \mathrm{~mL}$ 冰水中，立即有黄色固体析 出, 水洗, 乙醇重结晶, 得黄色固体 1-乙酰-2-硫代乙内 酰脲 $12.6 \mathrm{~g}$, 收率 80\%, m.p. 175 $177{ }^{\circ} \mathrm{C}$ (文献值 ${ }^{[7]}$ m.p. $\left.176 \sim 178{ }^{\circ} \mathrm{C}\right)$.

向 $250 \mathrm{~mL}$ 单口瓶中加入 $10 \mathrm{~g}(0.063 \mathrm{~mol}) 1$-乙酰-2硫代乙内酰脲和 $100 \mathrm{~mL}$ 浓盐酸，加热回流，1 h 后，反应 完全, 浓缩, 冷却, 析出固体, 抽滤, 水洗, 乙醇重结晶, 得黄色粒状固体 2-硫代乙内酰嫝 $6.5 \mathrm{~g}$, 收率 $80.5 \%$. m.p. $223 \sim 225{ }^{\circ} \mathrm{C}$ (文献值 ${ }^{[7]}$ m.p. $227{ }^{\circ} \mathrm{C}$ ); ${ }^{1} \mathrm{H}$ NMR $\left(\mathrm{DMSO}-d_{6}\right) \delta: 11.76(\mathrm{~s}, 1 \mathrm{H}), 9.85(\mathrm{~s}, 1 \mathrm{H}), 4.08(\mathrm{~s}, 2 \mathrm{H})$.

\subsubsection{5-亚环已基-2-硫代咪唑啉-4-酮(1)的合成}

向 $100 \mathrm{~mL}$ 单口瓶中加入 $4.64 \mathrm{~g}(0.04 \mathrm{~mol})$ 2-硫代乙 内酰脲, $4.5 \mathrm{~mL}(0.044 \mathrm{~mol})$ 环己酮, $3.3 \mathrm{~mL}(0.044 \mathrm{~mol}) 乙$ 醇胺和 $70 \mathrm{~mL}$ 无水乙醇，于室温下反应，5 h 后反应完全， 停止反应，抽滤，水洗，得淡黄色固体化合物 $16.4 \mathrm{~g}$, 收率 80.6\%. m.p. 260 261 ${ }^{\circ} \mathrm{C}$ (文献值 ${ }^{[8]}$ m.p. 263 265 $\left.{ }^{\circ} \mathrm{C}\right) ;{ }^{1} \mathrm{H}$ NMR (DMSO- $\left.d_{6}\right) \delta: 11.96(\mathrm{~s}, 1 \mathrm{H}), 11.52(\mathrm{~s}, 1 \mathrm{H})$, 2.83 (brs, 2H), 2.38 (brs, 2H), 1.56 (brs, 6H); IR v: 3445, 3142, 3002, 2940, 2858, 2773, 2548, 1826, 1730, 1658, $1541,1526 \mathrm{~cm}^{-1}$.

\subsubsection{5-亚环已基-2-甲硫基咪唑啉-4-酮(2)的合成}

向 $100 \mathrm{~mL}$ 单口瓶中加入 $50 \mathrm{~mL}$ 无水乙醇, $0.254 \mathrm{~g}$ (0.011 mol)钠，于室温下搅拌，待金属钠反应完全后， 分别加入 $1.960 \mathrm{~g}(0.010 \mathrm{~mol})$ 化合物 1 和 $0.68 \mathrm{~mL}(0.011$ $\mathrm{mol}$ )碘甲烷. $2 \mathrm{~h}$ 后反应完全，停止反应，将体系旋蒸至 近干，抽滤，水洗，得白色固体化合物 $21.736 \mathrm{~g}$, 收率 $82.7 \%$. m.p. $178 \sim 180{ }^{\circ} \mathrm{C}$ (文献值 ${ }^{[9]}$ m.p. $178 \sim 180{ }^{\circ} \mathrm{C}$ ); ${ }^{1} \mathrm{H}$ NMR $\left(\mathrm{CDCl}_{3}\right) \delta: 9.80$ (brs, $\left.1 \mathrm{H}\right), 3.00(\mathrm{t}, J=6.0 \mathrm{~Hz}$, 2H), 2.73 (t, $J=6.0 \mathrm{~Hz}, 2 \mathrm{H}), 2.60(\mathrm{~s}, 3 \mathrm{H}), 1.78 \sim 1.61(\mathrm{~m}$, $6 \mathrm{H})$; IR $v$ : 3358, 3123, 3063, 3033, 2933, 2847, 1693, $1635,1531 \mathrm{~cm}^{-1}$.

\subsection{4 目标化合物 $3 \mathbf{a} \sim \mathbf{3 r}$ 的合成通法}

向 $50 \mathrm{~mL}$ 三口瓶中加入 $1 \mathrm{mmol}$ 化合物 2,1 $\mathrm{mmol}$ 相应的苯胺或苠胺以及 $15 \mathrm{~mL}$ 冰醋酸, 加热至回流, 约 5 7 $\mathrm{h}$ 后反应达到平衡. 停止反应，旋蒸除去溶剂，分 别加入乙酸乙酯和石油醚混合溶剂, 有固体析出. 抽滤 后的固体经重结晶或硅胶柱层析分离, 得相应的目标化 合物 $3 \mathbf{a} \sim 3 \mathbf{r}$. 目标化合物的物理性质及结构表征数据 见表 1 、表 2 . 
表 1 目标化合物 3a 3r 的物理性质、收率及纯化方法

Table 1 Physical properties, yields and purification methods of compounds $\mathbf{3 a} \sim \mathbf{3 r}$

\begin{tabular}{|c|c|c|c|c|c|c|c|c|c|}
\hline \multirow{2}{*}{ Compd. } & \multirow{2}{*}{$n$} & \multirow{2}{*}{$\mathrm{R}^{1}$} & \multirow{2}{*}{$\mathrm{R}^{2}$} & \multirow{2}{*}{ 收率/\% } & \multirow{2}{*}{ 熔点 $/{ }^{\circ} \mathrm{C}$} & \multirow{2}{*}{ 外观 } & \multirow{2}{*}{ 纯化方法 } & \multicolumn{2}{|c|}{ HR-ESI-MS $m / z$} \\
\hline & & & & & & & & Calcd & Found \\
\hline $3 a$ & 0 & $\mathrm{CH}_{3}$ & $\mathrm{H}$ & 45.4 & $140 \sim 142$ & 白色固体 & 柱层析分离 & $270.1606[\mathrm{M}+\mathrm{H}]^{+}$ & 270.1609 \\
\hline $\mathbf{3 b}$ & 0 & $\mathrm{H}$ & $2-\mathrm{OCH}_{3}$ & 39.3 & $198 \sim 200$ & 黄色固体 & 柱层析分离 & $286.1556[\mathrm{M}+\mathrm{H}]^{+}$ & 286.1563 \\
\hline $3 c$ & 0 & $\mathrm{H}$ & $4-\mathrm{OCH}_{3}$ & 46.3 & $241 \sim 243$ & 黄色固体 & 甲醇重结晶 & $286.1556[\mathrm{M}+\mathrm{H}]^{+}$ & 286.1559 \\
\hline 3d & 0 & $\mathrm{H}$ & $2-\mathrm{Cl}$ & 39.8 & $217 \sim 219$ & 淡黄色固体 & 柱层析分离 & $288.0904[\mathrm{M}-\mathrm{H}]^{-}$ & 288.0911 \\
\hline $3 e$ & 0 & $\mathrm{H}$ & $3-\mathrm{Cl}$ & 34.3 & $272 \sim 274$ & 黄褐色固体 & 冰醋酸重结晶 & $288.0904[\mathrm{M}-\mathrm{H}]^{-}$ & 288.0908 \\
\hline $3 f$ & 0 & $\mathrm{H}$ & $4-\mathrm{Cl}$ & 29.2 & $247 \sim 249$ & 橙红色固体 & $\mathrm{DMF} /$ 水重结晶 & $288.0904[\mathrm{M}-\mathrm{H}]^{-}$ & 288.0917 \\
\hline $3 g$ & 0 & $\mathrm{H}$ & $2-\mathrm{F}$ & 30.4 & $226 \sim 228$ & 黄色固体 & 柱层析分离 & $272.1199[\mathrm{M}-\mathrm{H}]^{-}$ & 272.1189 \\
\hline $3 \mathrm{~h}$ & 0 & $\mathrm{H}$ & $4-\mathrm{F}$ & 59.7 & $264 \sim 266$ & 橙红色固体 & $\mathrm{DMF} /$ 水重结晶 & $272.1199[\mathrm{M}-\mathrm{H}]^{-}$ & 272.1193 \\
\hline $3 \mathbf{i}$ & 0 & $\mathrm{H}$ & $2,4-\mathrm{Cl}_{2}$ & 28.1 & $234 \sim 236$ & 白色固体 & 柱层析分离 & $323.0514[\mathrm{M}-\mathrm{H}]^{-}$ & 323.0509 \\
\hline $\mathbf{3 j}$ & 0 & $\mathrm{H}$ & $2-\mathrm{CH}_{3}$ & 26.8 & $235 \sim 237$ & 淡黄色固体 & 柱层析分离 & $270.1606[\mathrm{M}+\mathrm{H}]^{+}$ & 270.1615 \\
\hline $3 \mathbf{k}$ & 0 & $\mathrm{H}$ & $4-\mathrm{CH}_{3}$ & 71.6 & $245 \sim 247$ & 白色固体 & $\mathrm{DMF} /$ 水重结晶 & $270.1606[\mathrm{M}+\mathrm{H}]^{+}$ & 270.1605 \\
\hline 31 & 0 & $\mathrm{H}$ & $\mathrm{H}$ & 51.8 & $252 \sim 254$ & 黄色固体 & $\mathrm{DMF} /$ 水重结晶 & $254.1293[\mathrm{M}-\mathrm{H}]^{-}$ & 254.1299 \\
\hline $3 m$ & 1 & $\mathrm{H}$ & $\mathrm{H}$ & 56.9 & $218 \sim 220$ & 黄色固体 & 甲醇重结晶 & $270.1606[\mathrm{M}+\mathrm{H}]^{+}$ & 270.1618 \\
\hline $3 n$ & 1 & $\mathrm{H}$ & $2-\mathrm{Cl}$ & 58.4 & $189 \sim 191$ & 黄色固体 & 甲醇重结晶 & $302.1060[\mathrm{M}-\mathrm{H}]^{-}$ & 302.1054 \\
\hline 30 & 1 & $\mathrm{H}$ & $2-\mathrm{OCH}_{3}$ & 45.8 & $196 \sim 198$ & 白色固体 & 柱层析分离 & $298.1556[\mathrm{M}-\mathrm{H}]^{-}$ & 298.1563 \\
\hline $3 p$ & 1 & $\mathrm{H}$ & $4-\mathrm{F}$ & 47.0 & $209 \sim 211$ & 浅棕色固体 & 甲醇重结晶 & $286.1356[\mathrm{M}-\mathrm{H}]^{-}$ & 286.1348 \\
\hline $3 q$ & 1 & $\mathrm{H}$ & $4-\mathrm{Cl}$ & 55.8 & $219 \sim 221$ & 浅棕色固体 & 甲醇重结晶 & $302.1060[\mathrm{M}-\mathrm{H}]^{-}$ & 302.1065 \\
\hline $3 r$ & 1 & $\mathrm{H}$ & $4-\mathrm{OCH}_{3}$ & 56.2 & $200 \sim 202$ & 淡黄色固体 & 柱层析分离 & $298.1556[\mathrm{M}-\mathrm{H}]^{-}$ & 298.1551 \\
\hline
\end{tabular}

表 2 目标化合物 $\mathbf{3 a} \sim 3 \mathbf{r}$ 的 IR 及 ${ }^{1} \mathrm{H} N M R$ 数据

Table 2 The IR and ${ }^{1} \mathrm{H}$ NMR spectral data of compounds $\mathbf{3 a} \sim \mathbf{3 r}$

\begin{tabular}{|c|c|c|}
\hline Compd & $\mathrm{IR} / \mathrm{cm}^{-1}$ & ${ }^{1} \mathrm{H}$ NMR $\left(\mathrm{DMSO}-d_{6}\right) \delta$ \\
\hline 3a & $\begin{array}{l}3425,3211,3064,2924,2583 \\
1689,1644,1601,1572,1498\end{array}$ & $\begin{array}{l}10.69(\mathrm{~s}, 0.55 \mathrm{H}), 9.10(\mathrm{~s}, 0.41 \mathrm{H}), 7.47 \sim 7.19(\mathrm{~m}, 5 \mathrm{H}), 3.32(\mathrm{~s}, 3 \mathrm{H}), 2.90(\mathrm{brs}, 2 \mathrm{H}), 2.45(\mathrm{~s}, \\
1 \mathrm{H}), 2.27(\mathrm{brs}, 1 \mathrm{H}), 1.55(\mathrm{brs}, 6 \mathrm{H})\end{array}$ \\
\hline $3 \mathbf{b}$ & $\begin{array}{l}3231,3050,2928,2851,1692 \\
1652,1629,1584,1530\end{array}$ & $\begin{array}{l}10.03,8.48,8.25(\mathrm{brs}, 2 \mathrm{H}), 7.04 \sim 6.92(\mathrm{~m}, 4 \mathrm{H}), 3.88(\mathrm{~s}, 3 \mathrm{H}), 2.92(\mathrm{brs}, 2 \mathrm{H}), 2.58 \text { (brs, } 1 \mathrm{H}) \text {, } \\
2.19(\mathrm{brs}, 1 \mathrm{H}), 1.57 \text { (brs, } 6 \mathrm{H})\end{array}$ \\
\hline $3 c$ & $\begin{array}{l}3433,3050,2931,2855,2835 \\
1684,1663,1618,1589,1530\end{array}$ & $\begin{array}{l}10.27,9.27,9.15(\mathrm{brs}, 2 \mathrm{H}), 7.61 \sim 6.87(\mathrm{~m}, 4 \mathrm{H}), 3.71(\mathrm{~s}, 3 \mathrm{H}), 2.90(\mathrm{brs}, 2 \mathrm{H}), 2.54 \text { (brs, } 1 \mathrm{H}) \\
2.23(\mathrm{brs}, 1 \mathrm{H}), 1.57(\mathrm{brs}, 6 \mathrm{H})\end{array}$ \\
\hline 3d & $\begin{array}{l}3121,3053,2928,2853,1729 \\
1684,1660,1610,1572,1539\end{array}$ & $\begin{array}{l}11.95,11.52,10.55(\mathrm{brs}, 2 \mathrm{H}), 7.42 \sim 6.97(\mathrm{~m}, 4 \mathrm{H}), 2.86 \text { (brs, } 2 \mathrm{H}), 2.38 \text { (brs, } 2 \mathrm{H}), 1.55 \text { (brs, } \\
6 \mathrm{H})\end{array}$ \\
\hline $3 e$ & $\begin{array}{l}3436,3037,2931,2858,1687 \\
1659,1632,1601,1567,1528\end{array}$ & $\begin{array}{l}10.51,7.90,7.51(\mathrm{brs}, 2 \mathrm{H}), 7.33 \sim 7.02(\mathrm{~m}, 4 \mathrm{H}), 2.91 \text { (brs, } 2 \mathrm{H}), 2.52 \text { (brs, } 1 \mathrm{H}), 2.28 \text { (brs, } \\
1 \mathrm{H}), 1.58(\mathrm{brs}, 6 \mathrm{H})\end{array}$ \\
\hline $3 f$ & $\begin{array}{l}3391,3038,2930,2857,1667 \\
1645,1603,1574,1508\end{array}$ & $\begin{array}{l}10.42,9.30(\text { brs, } 2 \mathrm{H}), 7.69 \sim 7.32(\mathrm{~m}, 4 \mathrm{H}), 2.90(\text { brs, } 2 \mathrm{H}), 2.55 \text { (brs, } 1 \mathrm{H}), 2.25 \text { (brs, } 1 \mathrm{H}) \text {, } \\
1.57 \text { (brs, } 6 \mathrm{H})\end{array}$ \\
\hline $3 g$ & $\begin{array}{l}3447,3094,2937,2855,1693 \\
1655,1640,1613,1577,1555\end{array}$ & $\begin{array}{l}10.15,9.15,7.98(\mathrm{brs}, 2 \mathrm{H}), 7.57 \sim 7.01(\mathrm{~m}, 4 \mathrm{H}), 2.88 \text { (brs, } 2 \mathrm{H}), 2.46 \text { (brs, 1H), } 2.27 \text { (brs, } \\
1 \mathrm{H}), 1.56(\mathrm{brs}, 6 \mathrm{H})\end{array}$ \\
\hline $3 \mathbf{h}$ & $\begin{array}{l}3392,3068,2937,2843,1689 \\
1662,1594,1508\end{array}$ & $\begin{array}{l}10.39,9.49,9.35 \text { (brs, } 2 \mathrm{H}), 7.69 \sim 7.11(\mathrm{~m}, 4 \mathrm{H}), 2.90 \text { (brs, } 2 \mathrm{H}), 2.52 \text { (brs, 1H), } 2.20 \text { (brs, } \\
1 \mathrm{H}), 1.57 \text { (brs, } 6 \mathrm{H})\end{array}$ \\
\hline $3 \mathbf{i}$ & $\begin{array}{l}3404,3129,3026,2930,2854 \\
1728,1687,1659,1596,1538\end{array}$ & $\begin{array}{l}10.95,9.49,8.64 \text { (brs, } 2 \mathrm{H}), 7.61 \sim 7.06(\mathrm{~m}, 3 \mathrm{H}), 2.84 \text { (brs, } 2 \mathrm{H}), 2.52 \text { (brs, 1H), } 2.27 \text { (brs, } \\
1 \mathrm{H}), 1.54 \text { (brs, } 6 \mathrm{H})\end{array}$ \\
\hline $3 \mathbf{j}$ & $\begin{array}{l}3429,3121,3058,2929,2854 \\
1715,1691,1652,1597,1553\end{array}$ & $\begin{array}{l}\text { 10.24, } 9.26,7.54 \text { (brs, } 2 \mathrm{H}), 7.41 \sim 7.09(\mathrm{~m}, 4 \mathrm{H}), 2.89 \text { (brs, } 2 \mathrm{H}), 2.55 \text { (brs, 1H), } 2.24 \text { (brs, } \\
1 \mathrm{H}), 1.57 \text { (brs, } 6 \mathrm{H})\end{array}$ \\
\hline $3 \mathbf{k}$ & $\begin{array}{l}3420,3156,3039,2936,2855 \\
1687,1663,1632,1582,1515\end{array}$ & $\begin{array}{l}\text { 10.17, } 9.18,7.72(\mathrm{brs}, 2 \mathrm{H}), 7.35 \sim 6.97(\mathrm{~m}, 4 \mathrm{H}), 2.89 \text { (brs, } 2 \mathrm{H}), 2.42 \text { (brs, } 1 \mathrm{H}), 2.21 \text { (brs, } \\
4 \mathrm{H}), 1.55 \text { (brs, } 6 \mathrm{H})\end{array}$ \\
\hline 31 & $\begin{array}{l}3435,3037,2929,2857,1687 \\
1660,1582,1566,1527\end{array}$ & $\begin{array}{l}10.28,9.36,8.29 \text { (brs, } 2 \mathrm{H}), 7.69 \sim 6.92(\mathrm{~m}, 5 \mathrm{H}), 2.91 \text { (brs, } 2 \mathrm{H}), 2.56 \text { (brs, } 1 \mathrm{H}), 2.23 \text { (brs, } \\
1 \mathrm{H}), 1.58 \text { (brs, } 6 \mathrm{H})\end{array}$ \\
\hline $3 m$ & $\begin{array}{l}3282,3147,3064,2921,2850 \\
1691,1657,1619,1524\end{array}$ & $\begin{array}{l}\text { 10.46, 9.39, } 7.57 \text { (brs, } 2 \mathrm{H}), 7.36 \sim 7.22(\mathrm{~m}, 5 \mathrm{H}), 4.46 \text { (brs, } 2 \mathrm{H}), 2.88 \text { (brs, } 2 \mathrm{H}), 2.37 \sim 2.23 \\
\text { (brs, } 2 \mathrm{H}), 1.54 \text { (brs, } 6 \mathrm{H})\end{array}$ \\
\hline
\end{tabular}




\begin{tabular}{|c|c|c|}
\hline Compd. & $\mathrm{IR} / \mathrm{cm}^{-1}$ & ${ }^{1} \mathrm{H}$ NMR $\left(\mathrm{DMSO}-d_{6}\right) \delta$ \\
\hline $3 n$ & $\begin{array}{l}3270,3156,3067,2929,2854 \\
1688,1657,1617,1574,1529\end{array}$ & $\begin{array}{l}10.44,9.48,7.57(\text { brs, } 2 \mathrm{H}), 7.47 \sim 7.27(\mathrm{~m}, 4 \mathrm{H}), 4.54 \text { (brs, } 2 \mathrm{H}), 2.86 \text { (brs, } 2 \mathrm{H}) \text {, } \\
2.41 \text { (brs, } 1 \mathrm{H}), 2.21 \text { (brs, } 1 \mathrm{H}), 1.53(\text { brs, } 6 \mathrm{H})\end{array}$ \\
\hline 30 & $\begin{array}{l}3405,3166,3054,2927,2854 \\
1696,1660,1620,1531\end{array}$ & $\begin{array}{l}10.27,9.30(\text { brs, } 2 \mathrm{H}), 7.26 \sim 6.77(\mathrm{~m}, 4 \mathrm{H}), 4.44(\mathrm{brs}, 2 \mathrm{H}), 3.82(\mathrm{~s}, 3 \mathrm{H}), 2.86 \text { (brs, } \\
2 \mathrm{H}), 2.43(\text { brs, } 1 \mathrm{H}), 2.19(\mathrm{brs}, 1 \mathrm{H}), 1.53 \text { (brs, } 6 \mathrm{H})\end{array}$ \\
\hline $3 \mathbf{p}$ & $\begin{array}{l}3415,3253,3156,3059,2929 \\
2855,1693,1659,1602,1511\end{array}$ & $\begin{array}{l}10.48,9.56,7.75(\text { brs, } 2 \mathrm{H}), 7.37 \sim 7.13(\mathrm{~m}, 4 \mathrm{H}), 4.45 \text { (brs, } 2 \mathrm{H}), 2.87 \text { (brs, } 2 \mathrm{H}) \text {, } \\
2.44 \text { (brs, } 1 \mathrm{H}), 2.21 \text { (brs, } 1 \mathrm{H}), 1.53(\text { brs, } 6 \mathrm{H})\end{array}$ \\
\hline $3 q$ & $\begin{array}{l}3521,3247,3161,3051,2928 \\
1686,1659,1634,1580,1530\end{array}$ & $\begin{array}{l}10.42,9.44,7.61(\text { brs, } 2 \mathrm{H}), 7.41 \sim 7.30(\mathrm{~m}, 4 \mathrm{H}), 4.46 \text { (brs, } 2 \mathrm{H}), 2.86(\text { brs, } 2 \mathrm{H}) \text {, } \\
2.44 \text { (brs, } 1 \mathrm{H}), 2.21 \text { (brs, } 1 \mathrm{H}), 1.53(\text { brs, } 6 \mathrm{H})\end{array}$ \\
\hline $3 r$ & $\begin{array}{l}3279,3147,3064,2924,2852, \\
1695,1657,1616,1515\end{array}$ & $\begin{array}{l}10.37,9.31,7.49 \text { (brs, } 2 \mathrm{H}), 7.24 \sim 6.88(\mathrm{~m}, 4 \mathrm{H}), 4.40 \text { (brs, } 2 \mathrm{H}), 3.73(\mathrm{~s}, 3 \mathrm{H}), 2.87 \\
(\mathrm{brs}, 2 \mathrm{H}), 2.46 \text { (brs, } 1 \mathrm{H}), 2.19 \text { (brs, } 1 \mathrm{H}), 1.54(\text { brs, } 6 \mathrm{H})\end{array}$ \\
\hline
\end{tabular}

\section{3 化合物 $3 q$ 的单晶衍射}

将 $30 \mathrm{mg}$ 高纯度的化合物 $\mathbf{3 q}$ 置于 $50 \mathrm{~mL}$ 三角瓶中, 用甲醇加热将其溶解, 待固体溶解完全后再加入少量乙 酸乙酯, 用保鲜膜将瓶口封好, 并扎 3 5 个针孔, 置于 阴凉处, 让溶剂自然挥发, 大约一个星期瓶中长出符合 单晶衍射所需的晶体. 在低温条件 [173(2) K]下, 选取 $0.40 \mathrm{~mm} \times 0.25 \mathrm{~mm} \times 0.24 \mathrm{~mm}$ 的晶体置于四圆衍射仪 中, 以 $\mathrm{Mo} \mathrm{K \alpha}$ 射线为辐射源, 石墨单色器, 用 Xscan 程 序收集数据, 采用 $\omega-2 \theta$ 扫描方式, 在 $1.68^{\circ}<\theta<27.48^{\circ}$ 范围内收集到 8206 个衍射点, 其中 4011 个独立衍射点, $R(\mathrm{int})=0.0576$. 强度数据经半经验吸收校正, 在微机上 使用 SHELXTL-97 程序, 由直接法解出结构, 采用全矩 阵最小二乘法进行结构精修, 得到化合物 $\mathbf{3 q}$ 的晶体结 构如图 1 所示. 化合物 $\mathbf{3 q}$ 结构参数: $M_{\mathrm{r}}=335.83$, $\mathrm{C}_{17} \mathrm{H}_{22} \mathrm{ClN}_{3} \mathrm{O}_{2}$, Monoclinic, $P 2(1) / n,-7 \leqslant h \leqslant 7,-15 \leqslant$ $k \leqslant 15,-31 \leqslant l \leqslant 18, a=0.59895(12) \mathrm{nm}, \quad b=1.2161(2)$ $\mathrm{nm}, c=2.4289(5) \mathrm{nm}, \beta=94.03(3)^{\circ}, V=1.7646(6) \mathrm{nm}^{3}$, $D_{\mathrm{c}}=1.264 \mathrm{~g} / \mathrm{cm}^{3}, F(000)=712, Z=4, S=1.111, R_{1}=$ $0.0605, w R_{2}=0.1418[I>2 \sigma(I)], R_{1}=0.0735, w R_{2}=$ 0.1500 [all data]. 化合物 $\mathbf{3 q}$ 的晶体结构数据已存于剑桥 晶体结构数据库中, CCDC 号为 878441 .

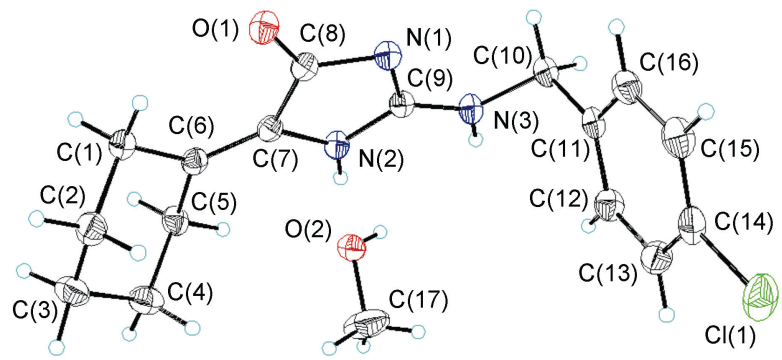

图 1 化合物 3q 的晶体结构

Figure 1 X-ray structure of compound $\mathbf{3 q}$

\section{4 生物活性测定}

10 种植物病原菌由南开大学元素有机化学国家重
点实验室分离保存. 用分析天平称取一定量的原药，加 DMSO 溶解，定容制备成浓度为 $5000 \mu \mathrm{g} / \mathrm{mL}$ 的溶液，然 后再制备成 $50 \mu \mathrm{g} / \mathrm{mL}$ 的含毒马铃薯葡萄糖琼脂培养基 (PDA)平板供试验用. 采用菌丝生长速率法 ${ }^{[6]}$, 以 $\mathrm{PDA}$ 培养基在平皿中培养测定目标化合物的杀菌活性,将培 养好的各种病原菌, 在无菌操作条件下用直径 $5 \mathrm{~mm}$ 的 灭菌打孔器, 自菌落边缘切取菌饼, 用接种器将菌饼接 种于含药平板中央，菌丝面向下，盖上㿼盖，置于 25 ${ }^{\circ} \mathrm{C}$ 培养箱中培养，根据空白对照培养血中菌落的生长情 况调查病原菌菌丝生长情况, 待空白对照中的菌落充分 生长后, 以十字交叉法测量各处理的菌落直径, 每个样 品平行测定 3 次, 取其平均值, 以百菌清和多菌灵为阳 性对照药剂. 以空白对照菌落增长直径和药剂处理的菌 落增长直径的差值与空白对照菌落增长直径的比值来 计算各药剂处理对各种病原菌的菌丝生长抑制率，化合 物 3 的杀菌活性测定结果见表 3.

\section{2 结果与讨论}

中间体 $\mathbf{1}$ 和 $\mathbf{2}$ 的合成易于进行收率较高，但是目标 化合物 3 的合成中由于反应达到平衡, 分离纯化后收率 $27 \% \sim 72 \%$ 之间并不理想. 近年来，国内外先后有文献 报道了 2-氨基-4-咪唑啉酮的结构类似物如 2-氨基-4-噻 唑啉酮类化合物存在互变异构现象，如化合物 4-1 和 4-2, 经过 NMR, $X$ 射线衍射方法等证实了该类化合物 的互变异构现象 ${ }^{[10 ~ 12]}$. 本文合成的该类化合物在它们 的 ${ }^{1} \mathrm{H}$ NMR 谱中, 氮原子上的两个活泼质子在多个化学 位移处以宽峰形式出现，而芳环以及环己酮环上的质子 也大多数呈现出宽单峰看不到明显的偶合裂分, 说明化 合物 3 的化学结构也存在类似的互变结构现象, 为了进 一步确证哪一种结构是最稳定的化学结构, 选择目标化 合物 3q 进行了单晶 $X$ 射线衍射，图 1 的结果证明结构 3q-1 是晶体中最稳定的化学结构. 同时化合物 3q 的单 晶衍射试验数据显示三个 $\mathrm{C}(9)-\mathrm{N}$ 键键长介于 $0.132 \mathrm{~nm}$ 
表 3 化合物 3a $3 \mathbf{r}$ 的杀菌活性测试结果 $(50 \mu \mathrm{g} / \mathrm{mL})$

Table 3 The fungicidal activities of compounds $\mathbf{3 a} \sim \mathbf{3 r}(50 \mu \mathrm{g} / \mathrm{mL})$

\begin{tabular}{|c|c|c|c|c|c|c|c|c|c|c|}
\hline \multirow{2}{*}{ 化合物 } & \multicolumn{10}{|c|}{ 抑制率/\% } \\
\hline & 花生褐斑 & 小麦赤霉 & 马铃薯晚疫 & 黄瓜枯萎 & 苹果轮纹 & 番茄早疫 & 黄瓜灰霉 & 油菜菌核 & 水稻纹枯 & 辣椒疫霉 \\
\hline $3 a$ & 25.0 & 4.8 & 23.8 & 11.1 & 28.2 & 43.5 & 15.7 & 21.4 & 13.2 & 26.1 \\
\hline $3 \mathbf{b}$ & 8.3 & 19.0 & 19.0 & 11.1 & 48.7 & 43.5 & 25.5 & 8.9 & 10.5 & 26.1 \\
\hline $3 c$ & 8.3 & 9.5 & 9.5 & 22.2 & 51.3 & 52.2 & 29.4 & 25.0 & 6.6 & 30.4 \\
\hline 3d & 25.0 & 14.3 & 23.8 & 11.1 & 51.3 & 56.5 & 43.1 & 32.1 & 18.4 & 39.1 \\
\hline $3 e$ & 25.0 & 4.8 & 14.3 & 5.6 & 23.1 & 43.5 & 19.6 & 17.9 & 10.5 & 26.1 \\
\hline $3 f$ & 8.3 & 4.8 & 0.0 & 11.1 & 25.6 & 43.5 & 23.5 & 21.4 & 6.6 & 34.8 \\
\hline $3 g$ & 25.0 & 19.0 & 28.6 & 16.7 & 64.1 & 65.2 & 35.3 & 26.8 & 34.2 & 34.8 \\
\hline $3 \mathrm{~h}$ & 8.3 & 19.0 & 14.3 & 0.0 & 20.5 & 39.1 & 23.5 & 8.9 & 10.5 & 39.1 \\
\hline $3 \mathbf{i}$ & 50.0 & 14.3 & 23.8 & 22.2 & 12.8 & 47.8 & 52.9 & 48.2 & 36.8 & 30.4 \\
\hline $3 \mathbf{j}$ & 8.3 & 23.8 & 19.0 & 11.1 & 38.5 & 43.5 & 41.2 & 14.3 & 32.9 & 43.5 \\
\hline $3 k$ & 16.7 & 14.3 & 19.0 & 11.1 & 20.5 & 47.8 & 29.4 & 35.7 & 6.6 & 30.4 \\
\hline 31 & 16.7 & 0.0 & 19.0 & 11.1 & 33.3 & 43.5 & 25.5 & 26.8 & 13.2 & 21.7 \\
\hline $3 m$ & 8.3 & 9.5 & 23.8 & 16.7 & 20.5 & 39.1 & 41.2 & 23.2 & 13.2 & 34.8 \\
\hline $3 n$ & 16.7 & 9.5 & 33.3 & 16.7 & 38.5 & 47.8 & 39.2 & 14.3 & 19.7 & 52.2 \\
\hline 30 & 41.7 & 9.5 & 42.9 & 22.2 & 56.4 & 52.2 & 37.3 & 8.9 & 22.4 & 30.4 \\
\hline $3 p$ & 50.0 & 0.0 & 23.8 & 11.1 & 33.3 & 30.4 & 29.4 & 70.4 & 7.9 & 8.7 \\
\hline $3 q$ & 25.0 & 14.3 & 28.6 & 16.7 & 38.5 & 43.5 & 35.3 & 28.6 & 6.6 & 75.0 \\
\hline $3 r$ & 41.7 & 9.5 & 28.6 & 27.8 & 28.2 & 47.8 & 35.3 & 17.9 & 13.2 & 26.1 \\
\hline 百菌清 & 75.0 & 66.7 & 81.0 & 83.3 & 92.3 & 73.9 & 96.1 & 96.4 & 96.1 & 82.6 \\
\hline 多菌灵 & 8.3 & 100.0 & 100.0 & 100.0 & 97.4 & 43.5 & 9.8 & 100.0 & 100 & 26.1 \\
\hline
\end{tabular}<smiles>[R]c1ccc(/C=C2/S/C(=N/c3nnc(COc4cccc([R])c4)s3)NC2=O)cc1</smiles><smiles>[R]c1ccc(/C=C2/SC(Nc3cc(C(C)OC)c(O)cc3C)=NC2=O)cc1</smiles>

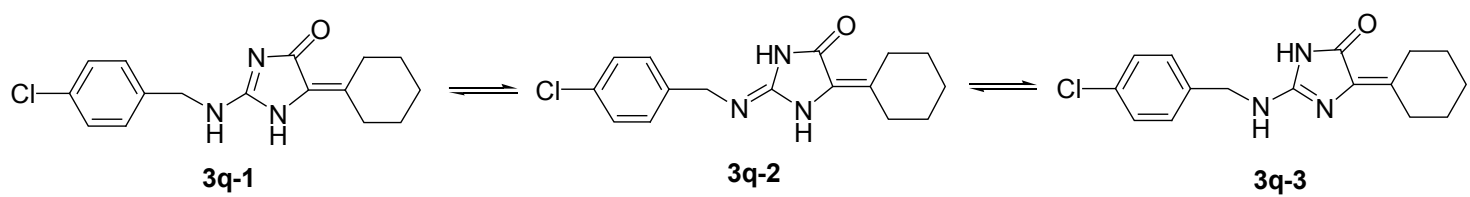

图 2 化合物 $\mathbf{3 q}$ 的互变异构

Figure 2 Tautomerism structure of compound $\mathbf{3 q}$

至 $0.135 \mathrm{~nm}$ 之间, 均较分子内其它 $\mathrm{C}-\mathrm{N}$ 单键键长 $(0.141 \sim 0.146 \mathrm{~nm})$ 要短, 由此可知 3 个 $\mathrm{C}(9)-\mathrm{N}$ 键在晶 体结构中均存在部分双键性质, 这也从另一个方面验证 了其互变异构体的存在, 与文献 $[13 \sim 14]$ 中通过 ${ }^{1} \mathrm{H}$ NMR 谱推断的结果一致. 图 1 的结果还显示, 在晶体中 存在一分子甲醇, $\mathrm{N}(2)-\mathrm{H}(2) \cdots \mathrm{O}(2)$ 间形成稳定的氢键, 说明甲醇分子在 3q 结晶时通过氢键帮助以 3q-1 的稳定 形式而存在, 量子化学计算结果也证明 3q-1 的能量是 最低的，结果将另文发表.

初步生物活性测定结果表明合成的目标化合物在 $50 \mu \mathrm{g} / \mathrm{mL}$ 浓度下对供试菌种均有一定的活性, 化合物 对苹果轮纹、番茄早疫、黄瓜灰霉、油菜菌核、辣椒疫 霉的抑制率高于对其他病原菌, 普遍低于对照药剂百菌 清和多菌灵, 但是 $\mathbf{3 p}$ 对油菜菌核的抑制率达 $70.4 \%, 3 q$
对辣椒疫霉的抑制率达 $75.0 \%$, 进一步测定结果显示 $3 p$ 对油菜菌核的 $\mathrm{EC}_{50}$ 为 $24.37 \mu \mathrm{g} / \mathrm{mL}, \mathbf{3 q}$ 对辣椒疫霉的 $\mathrm{EC}_{50}$ 为 $28.68 \mu \mathrm{g} / \mathrm{mL}$, 从结构上看芳环上含有氟或氯原 子的化合物杀菌活性较含有其它取代基的化合物好，这 为进一步的结构修饰提供了线索, 因此有必要对该类化 合物进行进一步的结构优化，以期发现杀菌活性更好的 化合物，相关研究正在进行中.

\section{References}

[1] Sobotka, W. Prog. Plant Protect. 2000, 40, 213.

[2] Lacroix, G.; Peignier, R.; Pepin, R.; Bascou, J.-P.; Perez, J.; Schmitz, C. US 6002016, 1999 [Chem. Abstr. 1999, 132, 35698].

[3] Ewa, S.; Katarzyna, K.; Anna, B.; Andrzej, K. Farmaco 2002, 57, 39.

[4] Ding, M. W.; Liu, Z. J.; Su, Y. L.; Zhu, L. CN 1508135, 2004 [Chem. Abstr. 2005, 143, 78187]. 
[5] Ding, M. W.; Liu, Z. J.; Sun, Y.; Zhu, L. CN 1496988, 2004 [Chem. Abstr. 2005, 143, 78184].

[6] Chen, S. C.; Zhang, C. Y.; Liu, J. P.; Wang, D. Q.; Wang, M. A. Chin. J. Org. Chem. 2010, 30, 1768 (in Chinese)

(陈守聪, 张春艳, 刘吉平, 王道全, 王明安, 有机化学, 2010, 30, 1768.)

[7] Johnson, T. B.; Nicolet, B. H. J. Am. Chem. Soc. 1911, 33, 1973.

[8] Yale, H. L. J. Am. Chem. Soc. 1953, 75, 675.

[9] Lei, J.P.; Dong, H. B.; Xu, Z. H.; Tang, B.; Wang, M. A. Chin. J. Pestic. Sci. 2012, 14, 445 (in Chinese).

(雷建平, 董宏波, 徐志红, 汤博, 王明安, 农药学学报, 2012, 14, 445.)
[10] Paola, V.; Athina, G.; Kitka, A.; Matteo, I.; Franca, Z. Bioorg. Med. Chem. 2006, 14, 3859.

[11] Ivanna, S.; Dmytro, A.; Ewa, S.; Katarzyna, K. K.; Borys, Z.; Olexandr, V.; Andrzej, G.; Roman, L. Bioorg. Med. Chem. 2010, 18,5090 .

[12] Wang, X. C.; Huang, G. L.; Quan, Z. J.; Yang, W. L. Chin. J. Org. Chem. 2008, 28, 870 (in Chinese).

(王喜存，黄国利，权正军，吕成伟，杨文龙，有机化学，2008， 28,870 .)

[13] Ewa, S.; Katarzyna, K. Farmaco 2002, 57, 355.

[14] Ewa, S.; Katarzyna, K. Farmaco 2002, 57, 909.

(Li, L.; Lu, Z.) 